\title{
Risk of myocardial infarction in men and women with type 2 diabetes in the UK: a cohort study using the General Practice Research Database
}

\author{
H. E. Mulnier • H. E. Seaman • V. S. Raleigh • \\ S. S. Soedamah-Muthu • H. M. Colhoun • \\ R. A. Lawrenson • C. S. de Vries
}

Received: 5 February 2008 / Accepted: 19 May 2008 / Published online: 26 June 2008

(C) Springer-Verlag 2008

\begin{abstract}
Aims/hypothesis Our primary aim was to establish reliable and generalisable estimates of the risk of myocardial infarction (MI) for men and women with type 2 diabetes in the UK compared with people without diabetes. Our secondary aim was to investigate how the MI risk associated with diabetes differs between men and women.
\end{abstract}

\author{
H. E. Mulnier $(\bowtie) \cdot$ H. E. Seaman • V. S. Raleigh • \\ R. A. Lawrenson \\ Postgraduate Medical School, University of Surrey, \\ Daphne Jackson Road, Manor Park, \\ Guildford, Surrey GU2 7WG, UK \\ e-mail: h.mulnier@surrey.ac.uk \\ V. S. Raleigh \\ Healthcare Commission, \\ London, UK
}

\section{S. S. Soedamah-Muthu $\cdot$ H. M. Colhoun}

Department of Epidemiology and Public Health,

Royal Free and University College London Medical School,

University College London Medical School,

London, UK

\section{S. S. Soedamah-Muthu}

Julius Center for Health Sciences and Primary Care,

University Medical Center Utrecht,

Utrecht, the Netherlands

H. M. Colhoun

Conway Institute of Biomolecular and Biomedical Research,

University College Dublin,

Dublin, Ireland

\section{R. A. Lawrenson}

Waikato Clinical School, University of Auckland,

Auckland, New Zealand

C. S. de Vries

Department of Pharmacy and Pharmacology, University of Bath,

Bath, UK
Methods A cohort study using the General Practice Research Database (1992-1999) was carried out, selecting 40,727 patients with type 2 diabetes and 194,913 age and sexmatched patients without diabetes. Rates of MI in men and women with and without diabetes were derived, as were hazard ratios for MI adjusted for known risk factors.

Results The rate of MI in men with type 2 diabetes was 19.74 (95\% CI 18.83-20.69) per 1,000 person-years compared with 16.18 (95\% CI 15.33-17.08) per 1,000 personyears in women with type 2 diabetes. The overall adjusted relative risk of $\mathrm{MI}$ in diabetes versus no diabetes was 2.13 (95\% CI 2.01-2.26) in men and 2.95 (95\% CI 2.75-3.17) in women and decreased with age in both sexes. Women with type 2 diabetes aged 35 to 54 years were at almost five times the risk of MI compared with women of the same age without diabetes (HR 4.86 [95\% CI 2.78-8.51]).

Conclusions/interpretation This study has demonstrated that women with type 2 diabetes are at a much greater relative risk of MI than men even when adjusted for risk factors.

Keywords Database - Myocardial infarction .

Primary care $\cdot$ Risk $\cdot$ Risk factors $\cdot$ Type 2 diabetes

\section{Abbreviations \\ GPRD General Practice Research Database \\ MI myocardial infarction}

\section{Introduction}

Although type 2 diabetes is known to be associated with an increased risk of myocardial infarction (MI) [1-4], there is still some discussion as to whether relative risk is higher in women with diabetes than in men with diabetes [5-8] and 
how risk is affected by increasing age [9]. Many published studies to date were either conducted in a population of one sex only $[2,4]$, included people with type 1 diabetes $[3,9$, 10] (which may affect the risk by age) or were limited to those under the age of $65[11,12]$ thereby excluding a measure of risk for patients with the highest prevalence of type 2 diabetes. In addition, many studies were based on comparatively small cohorts from populations with a high underlying risk of MI [13, 14] or were selected from secondary care [15] and consequently more likely to have worse disease progression. All of these limitations sometimes make it inappropriate to extrapolate rates and relative risks to other populations, and in particular to primary care, where the majority of decisions for early primary prevention in diabetes are made. Even results from meta-analyses are unclear. Huxley et al. reported an increased relative risk of MI in women with type 2 diabetes compared with no diabetes, but stated that their findings were limited by the small number of studies that presented data adjusted for relevant risk factors such as smoking, obesity and hypertension [5]. A further meta-analysis has shown an adjusted increased relative risk in women, but this was not statistically different from the increased relative risk in men [7].

The prevalence of diabetes is increasing $[16,17]$ and mortality rates in people with diabetes are continuing to rise, whereas they are decreasing in people without diabetes [18]. Reliable and generalisable data on the risk of MI in type 2 diabetes are essential, both for professionals responsible for primary prevention in type 2 diabetes and for health economists estimating the financial burden of type 2 diabetes on future health budgets. Current risk prediction calculators have been shown to be inaccurate in diabetes because they have been based on small numbers or groups that are not wholly representative of people with diabetes $[19,20]$. Others have stated that an ideal model would be based on a large-scale epidemiological study [21]. Our primary research question was to estimate the risk of MI associated with type 2 diabetes in a large unselected cohort drawn from the UK population. Our secondary research question was to investigate how the MI risk associated with diabetes differs between men and women.

\section{Methods}

Design We conducted a cohort study of MI risk in men and women with and without diabetes using a large, nationwide primary care database.

Data source The General Practice Research Database (GPRD) provides anonymised data from general practices across the UK. The GPRD is largely representative of the
UK general population in terms of age and sex distribution according to a comparison with Office for National Statistics data for 1994 projected from 1991 census data [22]. At any one time the GPRD population represents some $5 \%$ of the UK general population. In 1996, for example, $6.4 \%$ of the population in England was represented on the GPRD, compared with $5.1 \%$ in Wales, $2.8 \%$ in Scotland and 5.8\% in Northern Ireland $[22,23]$. The data stored on the GPRD include demographics, diagnoses and symptoms, prescriptions, hospital admission and hospital discharge information. Data on any patient's height, weight and smoking habit are also available although with some incompleteness that varies from practice to practice. Medical diagnoses and symptoms are recorded on the database using codes. GPRD data have been shown to be of high quality and validity [23-29] and have been used extensively for epidemiological research.

Study population We used the release of the database that was available at the University of Surrey at the time of this study and identified all men and women aged 35 to 89 on 1st January 1992 (baseline) in practices contributing research-quality data from baseline until 31st October 1999. Men and women with type 2 diabetes before baseline were identified using an algorithm based on age at diagnosis, type of treatment and other supporting evidence. Those with type 1 diabetes at any time were identified using a separate algorithm described elsewhere [30] and excluded from the study population. Full details of the cohort selection have been published elsewhere [30, 31]. For each patient with type 2 diabetes up to five comparison patients of the same age and sex and with no record of or treatment for diabetes at any time (i.e. before 1992 or during the follow-up period) were selected at random from the database.

Identifying MI events To ensure only first MI events were considered, all men and women with a history of MI or coronary re-vascularisation before baseline were excluded. All individuals with a first-ever diagnostic code for MI (e.g. 'myocardial infarction', 'heart attack') after baseline were then identified from within the study population. (A full list of codes is available on request.) Algorithms were established to assess the reliability of the MI diagnosis. The diagnosis was considered 'definite' when an MI was recorded and the patient died within 30 days or there was evidence of first treatment within 60 days with relevant prescribing (nitrates, beta blockers, calcium channel blockers, diuretics, ACE inhibitors, low-dose aspirin [see below], antiplatelet drugs or fibrinolytic drugs) plus other supporting evidence of MI within 60 days. Such supporting evidence included codes for ischaemic heart disease, location of infarct or confirmation of MI on ECG, raised 
cardiac enzymes, coronary artery re-vascularisation, cardiac rehabilitation, abnormal ECG or angiogram suggesting ischaemia, or hospital discharge letter. Patients with a 'probable' MI had a record of MI and relevant prescribing or supporting evidence, but not both. If only an MI record was evident, these were considered 'possible' MI cases. To test the sensitivity of the algorithm, the GPRD records for a random sample of patients with an MI were reviewed independently by two clinicians.

Other risk factors BMI was calculated using available height and weight recordings closest to baseline. For patients without height and weight records BMI was coded as 'unknown'. Patient records were viewed to confirm apparent BMIs of $<20 \mathrm{~kg} / \mathrm{m}^{2}$ or $>50 \mathrm{~kg} / \mathrm{m}^{2}$. BMIs of $<15 \mathrm{~kg} / \mathrm{m}^{2}$ or $>55 \mathrm{~kg} / \mathrm{m}^{2}$ ( $<1 \%$ of the recorded values) were deemed unreliable and were also coded as 'unknown'. Smoking status was also established according to the record closest to baseline and patients classified as 'smoker', 'exsmoker', 'non-smoker' or 'unknown'. GPRD smoking records may refer to the number of cigarettes smoked per day or to the smoking habit e.g. non-smoker, smoker. If the record closest to baseline indicated 'non-smoker' status, but a 'smoker' record existed earlier, the patient's smoking status was coded as 'ex-smoker'. Patients without records related to smoking habit were coded as 'unknown'. Among patients with diabetes, duration of diabetes at baseline was estimated either from the date of the first diabetes record or the date of the first diabetes-related prescription, whichever was earliest. If the date of diagnosis was considered to be unreliable due to the use of an improbable or impossible date $(3.2 \%)$, the average duration of diabetes within the patient's age-group was assumed for that patient and imputed. Patients with abnormal lipids (a record for raised cholesterol or dyslipidaemia or a pharmacological treatment related to cholesterol control) before baseline were identified. All patients with a diagnosis of hypertension before baseline were also identified. All identification of MI events and co-variates was performed 'blind' as to whether the patient had or did not have diabetes.

Statistical analysis Analyses were performed using Stata version 8 (Stata, College Station, TX, USA). Rates of MI were calculated for men and women with diabetes and the comparison group by age-group. Cox proportional hazards models were used to calculate HRs for MI in diabetes versus no diabetes and in men versus women both in patients with and without diabetes, stratified by age-group and adjusted for smoking, BMI, hypertension and abnormal lipids. Univariate and multivariate analyses were performed to assess the association between the risk factors and MI risk. To allow for attained age in these analyses, the date of origin was set to year of birth and the date of entry was 31
December 1991. To allow for change in age-group across the 7-year study period, all age-grouping was performed using Lexis expansion. Therefore a patient aged 64 years in 1992 who had an event at age 67 years would contribute to 1 year of follow-up in the 55- to 64-year-old age-group and to 3 years of follow-up in the 65- to 74-year-old age-group.

Sensitivity analyses Sensitivity analyses were carried out to test the reliability of the MI diagnosis. As low-dose aspirin is used widely in the primary prevention of MI, the effect of including or excluding aspirin as supporting evidence was investigated. Similarly, because any patient may be thought to have had an MI but later be diagnosed with ischaemic heart disease, we tested the algorithm by including and excluding codes for ischaemic heart disease from the supporting evidence criteria.

Ethics approval Approval for this study was given by the GPRD Scientific and Ethical Advisory Group.

\section{Results}

We identified 2,544 definite, 504 probable and 126 possible MI events among 40,727 men and women with type 2 diabetes and 4,948, 1,062 and 332 respectively in the comparison group of 194,913 people without diabetes. Table 1 provides the cohort characteristics at baseline and the percentages of patients with a first MI during follow-up. Sensitivity analysis including and excluding probable or possible MI events made no difference to the risk estimates. When a random sample of records from the definite and probable groups was examined in detail, no evidence to refute the diagnosis (e.g. a later diagnosis of epigastric pain and discontinuation of nitrates) could be found. As the number in the possible group was small and had little impact on the findings, we report results based on patients with a definite or probable MI only. The overall rate of MI in type 2 diabetic patients was 18.03 (95\% CI 17.41-18.69) per 1,000 person-years and 7.00 (95\% CI 6.82-7.18) per 1,000 person-years in the comparison group without diabetes (Table 2). The overall relative risk of $\mathrm{MI}$ in diabetes compared with no diabetes was 2.47 (95\% CI 2.36-2.59) adjusted for smoking habit, BMI, hypertension and abnormal lipids. This difference in risk decreased with increasing age (Table 3).

Rates of MI were higher in men than in women, both in type 2 diabetic patients and in the comparison group. The adjusted relative risk was higher in women than in men. Women aged 35 to 54 years with diabetes were at almost five times the risk of having an MI than their age-matched comparison group (HR 4.86 [95\% CI 2.78-8.51]), while the 
Table 1 Characteristics of the study cohort at baseline and number with MI events during the study

\begin{tabular}{|c|c|c|c|c|c|c|c|c|}
\hline & \multicolumn{4}{|c|}{ Type 2 diabetes } & \multicolumn{4}{|c|}{ Comparison group } \\
\hline & \multicolumn{2}{|l|}{ Men } & \multicolumn{2}{|l|}{ Women } & \multicolumn{2}{|l|}{ Men } & \multicolumn{2}{|l|}{ Women } \\
\hline & $n$ & $\%$ & $n$ & $\%$ & $n$ & $\%$ & $n$ & $\%$ \\
\hline Total & 21,165 & 52.0 & 19,562 & 48.0 & 99,658 & 51.1 & 95,255 & 48.9 \\
\hline \multicolumn{9}{|l|}{ Age (years) } \\
\hline $35-54$ & 3,868 & 18.3 & 2,474 & 12.7 & 19,086 & 19.2 & 12,341 & 13.0 \\
\hline $55-64$ & 5,681 & 26.8 & 4,339 & 22.2 & 27,170 & 27.3 & 21,451 & 22.5 \\
\hline $65-74$ & 6,664 & 31.5 & 6,003 & 30.7 & 30,827 & 30.9 & 29,230 & 30.7 \\
\hline $75-84$ & 4,291 & 20.3 & 5,412 & 27.7 & 19,556 & 19.6 & 25,892 & 27.2 \\
\hline$\geq 85$ & 661 & 3.1 & 1,334 & 6.8 & 3,019 & 3.0 & 6,341 & 6.7 \\
\hline Average age in 1992 (years) & 65.3 & & 68.5 & & 65.0 & & 68.3 & \\
\hline \multicolumn{9}{|l|}{ Smoking status } \\
\hline Current smoker & 4,309 & 20.4 & 2,412 & 12.33 & 20,137 & 20.21 & 12,672 & 13.3 \\
\hline Ex-smoker & 1,994 & 9.4 & 940 & 4.8 & 7,881 & 7.9 & 3,940 & 4.1 \\
\hline Non-smoker & 11,549 & 54.6 & 12,487 & 63.8 & 42,070 & 42.2 & 50,637 & 53.2 \\
\hline Unknown & 3,313 & 15.6 & 3,723 & 19.0 & 29,570 & 29.7 & 28,006 & 29.4 \\
\hline Hypertension & 6,085 & 28.8 & 7,579 & 38.7 & 15,028 & 15.1 & 19,609 & 20.6 \\
\hline Abnormal lipids & 1,344 & 4.6 & 1,270 & 4.9 & 2,905 & 2.1 & 2,582 & 2.0 \\
\hline \multicolumn{9}{|l|}{ Duration of diabetes (years) } \\
\hline $0-4$ & 10,650 & 50.3 & 9,761 & 49.9 & NA & & NA & \\
\hline $5-9$ & 4,872 & 23.0 & 4,668 & 23.9 & NA & & NA & \\
\hline $10-14$ & 2,488 & 11.8 & 2,377 & 12.2 & NA & & NA & \\
\hline$\geq 15$ & 2,483 & 11.7 & 2,139 & 10.9 & NA & & NA & \\
\hline Unknown & 672 & 3.2 & 617 & 3.2 & NA & & NA & \\
\hline \multicolumn{9}{|l|}{ BMI $\left(\mathrm{kg} / \mathrm{m}^{2}\right)$} \\
\hline $15-19$ & 198 & 0.9 & 400 & 2.0 & 1,539 & 1.5 & 2,888 & 3.0 \\
\hline $20-24$ & 3,966 & 18.7 & 3,020 & 15.4 & 18,972 & 19.0 & 20,268 & 21.3 \\
\hline $25-29$ & 7,620 & 36.0 & 4,837 & 24.7 & 26,803 & 26.9 & 19,824 & 20.8 \\
\hline $30-34$ & 2,961 & 14.0 & 3,087 & 15.8 & 6,322 & 6.3 & 6,688 & 7.0 \\
\hline$\geq 35$ & 919 & 4.3 & 1,964 & 10.0 & 1,057 & 1.1 & 2,389 & 2.5 \\
\hline Unknown & 5,501 & 26.0 & 6,254 & 32.0 & 44,965 & 45.1 & 43,198 & 45.4 \\
\hline \multicolumn{9}{|l|}{ MI after baseline } \\
\hline Definite & 1,435 & 6.8 & 1,109 & 5.7 & 3,108 & 3.1 & 1,840 & 1.9 \\
\hline Probable & 304 & 1.4 & 200 & 1.0 & 663 & 0.7 & 399 & 0.4 \\
\hline Possible & 71 & 0.3 & 55 & 0.3 & 184 & 0.2 & 148 & 0.2 \\
\hline
\end{tabular}

NA, not applicable

risk among men of that age-group with diabetes was double that of the comparison group (HR 2.21 [95\% CI 1.922.54]). When we assessed the effect of the risk factors on MI risk using univariate and multivariate analyses (data not shown), we found that no one risk factor accounted for the increased relative risk in women or the decrease in diabetes-related risk with increasing age. Stratification by duration of diabetes in 5 year bands revealed a similar relative risk pattern, with women in the youngest agegroups being at greatest relative risk. Patients with a longer duration of diabetes were at greater risk than those with short duration, but this was not significant across all the age groups.

Our examination of the effect of including or excluding aspirin as supporting evidence for a genuine first MI event revealed that all MI cases selected by aspirin prescriptions and an MI code had been prescribed aspirin for the treatment of ischaemic heart disease 60 days pre or post MI. Thus aspirin was included in the medication list to support the diagnosis of MI. The exclusion of cases with a code for MI and supporting evidence of a code for ischaemic heart disease with or without a pharmacological treatment, but no other supporting evidence $(0.4 \%$ of the diabetes cohort and $0.2 \%$ of the comparison group) made no difference to the risk estimates.

\section{Discussion}

This study has shown that in a cohort of people with no prior MI, men and women with type 2 diabetes in the UK are at more than twice the risk of MI than those without 
Table 2 Number of MI events and rates per 1,000 person-years by sex and attained age-group in patients with and without type 2 diabetes

\begin{tabular}{|c|c|c|c|c|c|c|c|}
\hline & \multicolumn{2}{|c|}{ Events $(n)$} & \multicolumn{2}{|c|}{ Person-years at risk } & \multicolumn{3}{|c|}{ Rate per 1,000 person-years $(95 \% \mathrm{CI})$} \\
\hline & Men & Women & Men & Women & All & Men & Women \\
\hline \multicolumn{8}{|c|}{ Patients with type 2 diabetes } \\
\hline Age $35-54$ years & 119 & 40 & 13,779 & 9,269 & $6.90(5.91-8.06)$ & $8.64(7.22-10.34)$ & $4.32(3.17-5.88)$ \\
\hline Age $55-64$ years & 328 & 177 & 23,374 & 17,181 & $12.45(11.41-13.59)$ & $14.03(12.59-15.64)$ & $10.30(8.89-11.94)$ \\
\hline Age $65-74$ years & 655 & 405 & 29,145 & 25,497 & $19.40(18.27-20.6)$ & $22.47(20.82-24.26)$ & $15.88(14.41-17.51)$ \\
\hline Age $75-84$ years & 517 & 517 & 18,135 & 22,242 & $25.61(24.1-27.22)$ & $28.51(26.15-31.08)$ & $23.24(21.32-25.34)$ \\
\hline Age $\geq 85$ years & 120 & 170 & 3,675 & 6,715 & $27.91(24.88-31.32)$ & $32.65(27.3-39.05)$ & $25.32(21.78-29.42)$ \\
\hline All ages & 1,739 & 1,309 & 88,108 & 80,904 & $18.03(17.41-18.69)$ & $19.74(18.83-20.69)$ & $16.18(15.33-17.08)$ \\
\hline \multicolumn{8}{|c|}{ Patients without type 2 diabetes } \\
\hline Age $35-54$ years & 157 & 25 & 69,571 & 45,526 & $1.58(1.37-1.83)$ & $2.26(1.93-2.64)$ & $0.55(0.37-0.81)$ \\
\hline Age $55-64$ years & 617 & 140 & 114,174 & 86,667 & $3.77(3.51-4.05)$ & $5.40(4.99-5.85)$ & $1.62(1.37-1.91)$ \\
\hline Age $65-74$ years & 1,367 & 563 & 141,726 & 132,834 & $7.03(6.72-7.35)$ & $9.65(9.15-10.17)$ & $4.24(3.90-4.60)$ \\
\hline Age $75-84$ years & 1,322 & 1,054 & 91,041 & 118,941 & $11.32(10.87-11.78)$ & $14.52(13.76-15.33)$ & $8.86(8.34-9.41)$ \\
\hline Age $\geq 85$ years & 308 & 457 & 19,496 & 38,665 & $13.15(12.25-14.12)$ & $15.80(14.13-17.67)$ & $11.82(10.78-12.96)$ \\
\hline All ages & 3,771 & 2,239 & 436,009 & 422,632 & $7.00(6.82-7.18)$ & $8.65(8.38-8.93)$ & $5.30(5.08-5.52)$ \\
\hline
\end{tabular}

diabetes, a difference that remains after allowing for ageing and adjusting for BMI, smoking, hypertension and abnormal lipids. The MI risk was consistently higher in men than in women for all age-groups, both in people with and without diabetes, although the relative risk of MI associated with diabetes was much greater in women than men. Younger women with type 2 diabetes carried a relative risk of MI that was five times greater than their comparison group without diabetes. This increased relative risk in women remained even when adjusted for risk factors and corroborates the findings of Huxley et al., which showed women to be at a greater relative risk than men [5]. This finding is consistent with our previous work on all-cause mortality and stroke in type 2 diabetes [31, 32]. We consider that the data presented here are consistent and that this pattern of increased relative risk in women is likely to be real.

The sensitivity and validity of the algorithm used to detect MI may be questioned. There was no evidence to dispute the diagnosis of MI in cases defined as definite or probable when the sample of complete GPRD records was examined. The rates of MI reported here are similar to those from other studies. For example in type 2 diabetes our rates for ages 65 to 84 years were 24.8 (95\% CI 23.4-26.2) per 1,000 person-years for men and 19.3 (95\% CI 18.1-20.6) per 1,000 person-years for women, compared with 32.9 (95\% CI 25.2-43.0) and 22.9 (95\% CI 16.7-31.2) in men and women respectively reported by McAlpine et al. [13] for the same age-group. Our rates were lower than those reported for diabetes in a Finnish study, where the underlying rate of MI is higher than that in the UK [33]. Our rates for men and women aged 50 to 64 years without diabetes were very similar to those reported for Oxfordshire in 1994 to 1995 [34] and slightly lower than those reported for Glasgow and Belfast in the late 1980s and early 1990s [35]. These comparisons demonstrate the robustness of these data and suggest (1) that they could be used as a baseline for risk of MI prior to the implementation of recent guidelines and targets [36-38] and (2) that they could be used for risk prediction calculations in type 2 diabetes.

Unlike other studies where the general population was used for comparison, this study compared MI risk in patients with type 2 diabetes and a comparison group that

Table 3 HRs (95\% CI) for MI in type 2 diabetes compared with no diabetes stratified by sex and attained age-group, adjusted for smoking habit, BMI, hypertension and abnormal lipids

\begin{tabular}{lllr}
\hline & All & Men & Women \\
\hline Diabetes $(n)$ & 40,727 & 21,165 & 19,562 \\
No diabetes $(n)$ & 194,913 & 99,658 & 95,255 \\
Age 35-54 years & $3.07(2.43-3.88)$ & $2.69(2.07-3.49)$ & $4.86(2.78-8.51)$ \\
Age 55-64 years & $2.83(2.51-3.19)$ & $2.21(1.92-2.54)$ & $5.25(4.11-6.69)$ \\
Age 65-74 years & $2.59(2.40-2.81)$ & $2.17(1.97-2.39)$ & $3.45(3.01-3.95)$ \\
Age $\geq 75$ years & $2.21(2.07-2.36)$ & $1.96(1.78-2.15)$ & $2.45(2.24-2.69)$ \\
All ages & $2.47(2.36-2.59)$ & $2.13(2.01-2.26)$ & $2.95(2.75-3.17)$ \\
\hline
\end{tabular}


remained diabetes-free throughout the follow-up period. According to Harris et al. [39] the average time from onset to diagnosis of type 2 diabetes has been estimated to be at least 4 to 7 years. The follow-up period of our study was up to 7 years, therefore all persons who were diagnosed in the 7 year follow-up period were excluded from the potential comparison group and consequently most patients with undiagnosed diabetes will have been excluded from the comparison group. The comparison group may still contain some patients with undiagnosed diabetes but their contribution to the risk estimates for the comparison group is likely to be minimal.

Various limitations to the data exist, which may leave the risk estimates susceptible to some residual confounding. Limitations to the data on duration of diabetes, BMI and smoking have been described previously [31, 32]. Extensive sensitivity analyses that tested the effect of including and excluding patients with unknown smoking status or BMI, as well as the effect of using status at baseline as opposed to the index date, have also been reported previously [32]. These analyses suggested that the impact of residual confounding on the risk estimates for diabetes and no diabetes will be minimal. In brief, the effect of the unknown risk factor data may be to slightly overestimate the reported risk attributed to diabetes. We have provided the numbers with unknown data in Table 1. We believe that to disregard these data completely could produce more greatly overestimated risk estimates for diabetes. We may have missed a small number of people who were treated for hypertension before baseline and who did not have a diagnosis of hypertension. To assess hypertension in all those people with hypertensive treatment only would have been impractical for this study. In addition, we believe the numbers would have been small and would have had little impact on our findings. We have not included measures of glycaemic control, because the follow-up period was largely before laboratory standardisation of glycated haemoglobin.

\section{Conclusions}

This study provides an estimate of the relative risk of MI for the UK population by age and sex for patients with and without type 2 diabetes. We have demonstrated that women are at a much greater relative risk than men, even when that risk is adjusted for the most influential risk factors. Given the increasing epidemic of type 2 diabetes and the falling average age at onset, plus the increased relative risk in the younger age-groups and an attributable risk of diabetes of some eleven MI cases per 1,000 person-years, the impact on the economic burden of healthcare from diabetes will be huge. Against this background, the results from this study could be useful ways of reinforcing and informing clinical decision-making especially in primary care.

Acknowledgements This study was funded by an unconditional research grant from the British Heart Foundation. The authors' work was entirely independent of the funding body.

Duality of interest H. M. Colhoun has received speaker and consultancy fees from Pfizer. All other authors declare that there is no duality of interest associated with this manuscript.

\section{Reference}

1. Kannel WB, McGee DL (1979) Diabetes and cardiovascular disease. The Framingham study. JAMA 241:2035-2038

2. Fuller JH, Shipley MJ, Rose G, Jarrett RJ, Keen H (1983) Mortality from coronary heart disease and stroke in relation to degree of glycaemia: the Whitehall study. Br Med J (Clin Res Ed) 287:867-870

3. Asia Pacific Cohort Studies Collaboration (2003) The effects of diabetes on the risks of major cardiovascular diseases and death in the Asia-Pacific Region. Diabetes Care 26:360-366

4. Stamler J, Vaccaro O, Neaton JD, Wentworth D (1993) Diabetes, other risk factors, and 12-yr cardiovascular mortality for men screened in the Multiple Risk Factor Intervention Trial. Diabetes Care 16:434-444

5. Huxley R, Barzi F, Woodward M (2006) Excess risk of fatal coronary heart disease associated with diabetes in men and women: meta-analysis of 37 prospective cohort studies. BMJ 332:73-78

6. Lee WL, Cheung AM, Cape D, Zinman B (2000) Impact of diabetes on coronary artery disease in women and men: a metaanalysis of prospective studies. Diabetes Care 23:962-968

7. Kanaya AM, Grady D, Barrett-Connor E (2002) Explaining the sex difference in coronary heart disease mortality among patients with type 2 diabetes mellitus: a meta-analysis. Arch Intern Med $162: 1737-1745$

8. Orchard TJ (1996) The impact of gender and general risk factors on the occurrence of atherosclerotic vascular disease in noninsulin-dependent diabetes mellitus. Ann Med 28:323-333

9. Booth GL, Kapral MK, Fung K, Tu JV (2006) Relation between age and cardiovascular disease in men and women with diabetes compared with non-diabetic people: a population-based retrospective cohort study. Lancet 368:29-36

10. Barrett-Connor E, Wingard DL (1983) Sex differential in ischemic heart disease mortality in diabetics: a prospective populationbased study. Am J Epidemiol 118:489-496

11. Jousilahti P, Vartiainen E, Tuomilehto J, Puska P (1999) Sex, age, cardiovascular risk factors, and coronary heart disease: a prospective follow-up study of 14,786 middle-aged men and women in Finland. Circulation 99:1165-1172

12. Niskanen L, Turpeinen A, Penttila I, Uusitupa MI (1998) Hyperglycemia and compositional lipoprotein abnormalities as predictors of cardiovascular mortality in type 2 diabetes: a 15-year follow-up from the time of diagnosis. Diabetes Care 21:1861-1869

13. McAlpine RR, Morris AD, Emslie-Smith A, James P, Evans JM (2005) The annual incidence of diabetic complications in a population of patients with type 1 and type 2 diabetes. Diabet Med 22:348-352

14. Laakso M, Ronnemaa T, Lehto S, Puukka P, Kallio V, Pyorala K (1995) Does NIDDM increase the risk for coronary heart disease similarly in both low- and high-risk populations? Diabetologia $38: 487-493$ 
15. Avogaro A, Giorda C, Maggini M et al (2007) Incidence of coronary heart disease in type 2 diabetic men and women: impact of microvascular complications, treatment, and geographic location. Diabetes Care 30:1241-1247

16. Amos AF, McCarty DJ, Zimmet P (1997) The rising global burden of diabetes and its complications: estimates and projections to the year 2010. Diabet Med 14(Suppl 5):S1-S85

17. King H, Aubert RE, Herman WH (1998) Global burden of diabetes, 1995-2025: prevalence, numerical estimates, and projections. Diabetes Care 21:1414-1431

18. Hu J, Robbins G, Ugnat AM, Waters C (2005) Trends in mortality from diabetes mellitus in Canada, 1986-2000. Chronic Dis Can 26:25-29

19. Coleman RL, Stevens RJ, Retnakaran R, Holman RR (2007) Framingham, SCORE, and DECODE risk equations do not provide reliable cardiovascular risk estimates in type 2 diabetes. Diabetes Care 30:1292-1293

20. Yeo WW, Yeo KR (2001) Predicting CHD risk in patients with diabetes mellitus. Diabet Med 18:341-344

21. Stevens RJ, Kothari V, Adler AI, Stratton IM, United Kingdom Prospective Diabetes Study (2001) The UKPDS risk engine: a model for the risk of coronary heart disease in Type II diabetes (UKPDS 56). Clin Sci 101:671-679

22. Lawson DH, Sherman V, Hollowell J (1998) The General Practice Research Database. Scientific and Ethical Advisory Group. QJM 91:445-452

23. Walley T, Mantgani A (1997) The UK General Practice Research Database. Lancet 350:1097-1099

24. No authors listed (2000) Key Health Statistics from General Practice 1998, Series MB6 No. 2. 2000. Office for National Statistics, London

25. Lawrenson R, Todd JC, Leydon GM, Williams TJ, Farmer RD (2000) Validation of the diagnosis of venous thromboembolism in general practice database studies. Br J Clin Pharmacol 49:591-596

26. Hollowell J (1997) The General Practice Research Database: quality of morbidity data. Popul Trends $87: 36-40$

27. Jick SS, Kaye JA, Vasilakis-Scaramozza C et al (2003) Validity of the general practice research database. Pharmacotherapy 23:686-689

28. Wood L, Martinez C (2004) The general practice research database: role in pharmacovigilance. Drug Safety 27:871-881
29. Garcia Rodriguez LA, Perez GS (1998) Use of the UK General Practice Research Database for pharmacoepidemiology. Br J Clin Pharmacol 45:419-425

30. Soedamah-Muthu SS, Fuller JH, Mulnier HE, Raleigh VS, Lawrenson RA, Colhoun HM (2006) All-cause mortality rates in patients with type 1 diabetes mellitus compared with a nondiabetic population from the UK general practice research database, 1992-1999. Diabetologia 49:660-666

31. Mulnier H, Seaman H, Raleigh V et al (2006) Mortality amongst people with type 2 diabetes in the UK. Diabet Med 23:516-521

32. Mulnier HE, Seaman HE, Raleigh VS et al (2006) Risk of stroke in people with type 2 diabetes in the UK: a study using the General Practice Research Database. Diabetologia 49:2859-2865

33. Juutilainen A, Kortelainen S, Lehto S, Ronnemaa T, Pyorala K, Laakso M (2004) Gender difference in the impact of type 2 diabetes on coronary heart disease risk. Diabetes Care 27:2898 2904

34. Volmink JA, Newton JN, Hicks NR, Sleight P, Fowler GH, Neil HAW (1998) Coronary event and case fatality rates in an English population: results of the Oxford myocardial infarction incidence study. Heart 80:40-44

35. Tunstall-Pedoe $\mathrm{H}$, Kuulasmaa $\mathrm{K}$, Mahonen $\mathrm{M}$, Tolonen $\mathrm{H}$, Ruokokoski E (1999) Contribution of trends in survival and coronary-event rates to changes in coronary heart disease mortality: 10-year results from 37 WHO MONICA Project populations. Lancet 353:1547-1557

36. British Cardiac Society, British Hypertension Society, Diabetes UK, HEART UK, Primary Care Cardiovascular Society, Stroke Association (2005) JBS 2: Joint British Societies' guidelines on prevention of cardiovascular disease in clinical practice. Heart 91 (Suppl 5):v1-v52

37. Department of Health (2001) National Service Framework for Diabetes. HMSO, London

38. Department of Health (2000) National Service Framework for Coronary Heart Disease: modern standards and service models. HMSO, London

39. Harris MI, Klein R, Welborn TA, Knuiman MW (1992) Onset of NIDDM occurs at least $4-7$ yr before clinical diagnosis. Diabetes Care 15:815-819 